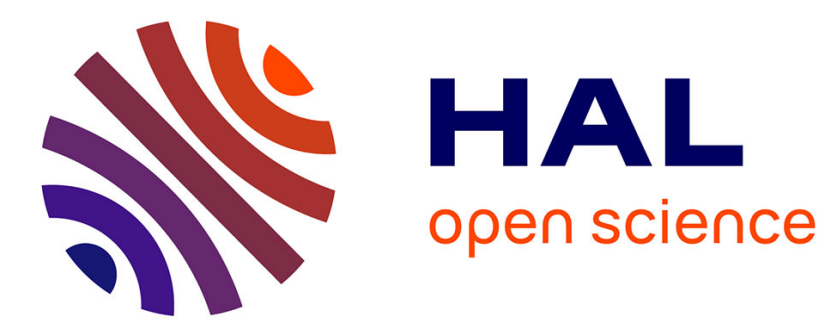

\title{
Étude des vibrations de la surface d'un cristal
}

\author{
P. Masri, L. Dobrzynski
}

\section{To cite this version:}

P. Masri, L. Dobrzynski. Étude des vibrations de la surface d'un cristal. Journal de Physique, 1971, 32 (4), pp.295-299. 10.1051/jphys:01971003204029500 . jpa-00207056

\section{HAL Id: jpa-00207056 https://hal.science/jpa-00207056}

Submitted on 1 Jan 1971

HAL is a multi-disciplinary open access archive for the deposit and dissemination of scientific research documents, whether they are published or not. The documents may come from teaching and research institutions in France or abroad, or from public or private research centers.
L'archive ouverte pluridisciplinaire HAL, est destinée au dépôt et à la diffusion de documents scientifiques de niveau recherche, publiés ou non, émanant des établissements d'enseignement et de recherche français ou étrangers, des laboratoires publics ou privés. 
Classification :

Physics Abstracts

16.20

\title{
ÉTUDE DES VIBRATIONS DE LA SURFACE D’UN CRISTAL
}

\author{
par P. MASRI (*) \\ Section d'Etudes des Interactions Gaz-Solides \\ Centre d'Etudes Nucléaires de Saclay \\ et L. DOBRZYNSKI (**) \\ Physique des Solides, I. S. E. N., 3, rue François Baës, 59, Lille
}

(Reçu le 3 novembre 1970)

\begin{abstract}
Résumé. - Nous étudions par la méthode des fonctions de Green les vibrations de la surface (001) d'un cristal cubique simple dans la direction de propagation [100]. Les constantes de force ont été choisies telles que le cristal soit isotrope à la limite des grandes longueurs d'onde. Ce modèle de cristal permet de retrouver les ondes de Rayleigh à la limite des grandes longueurs d'onde, ainsi que des états localisés en surface à l'intérieur du ( gap ) délimité par les bandes du cristal infini.

Nous avons calculé, tout au long de l'axe de symétrie $\Delta$ (Fig. 1), les états localisés en surface qui correspondent aux ondes de Rayleigh à la limite des grandes longueurs d'onde. Nous avons aussi déterminé la partie de l'axe $\Delta$ pour laquelle les états à l'intérieur du ( gap ) existent. Nous trouvons aussi deux ( antirésonances ) de surface dont l'une correspond à une très faible variation de la densité d'état alors que l'autre correspond à une forte variation de la densité d'état.

Abstract. - The vibrations of a (001) free surface of a simple cubic cristal for the [100] direction of propagation are studied using the Green's functions method. The force constants are chosen in order to have an isotropic crystal at the limit of long wavelengths. This crystal model, giving the classical Rayleigh waves at the long wavelength limit, allows to obtain surface states which lie in the gap between the bands of the bulk (infinite crystal) modes.

We calculated along the symmetry axis $\Delta$ (Fig. 1) the surface states corresponding to the Rayleigh waves in the long wavelength limit, and the extent of the $\Delta$ axis along which states exist in the gap. Two (" anti-resonant surface states ) are found corresponding respectively to a very small and to a large variation of the density of states.
\end{abstract}

I. Introduction. - Lifshitz et al. [1] ont proposé pour l'étude des vibrations des surfaces cristallines une méthode de résolution utilisant les fonctions de Green. Cette méthode appliquée par Rosenzweig [2] à un modèle de cristal cubique simple avecdes interactions centrales entre atomes premiers et seconds voisins, des forces dues à la rigidité angulaire d'un système de trois premiers voisins formant un angle droit à l'équilibre et des constantes de force telles que le cristal soit isotrope à la limite des grandes longueurs d'onde, lui a permis de retrouver à cette limite les ondes de Rayleigh pour une surface (001) et dans la direction de propagation [100].

Brown [3] utilisa cette méthode pour étudier les états liés et résonnants possibles de phonons et d'électrons créés par les surfaces et les dislocations.

Dobrzynski et Leman [4] ont montré comment cette méthode peut être simplifiée dans les calculs

(*) Services de Physique Générale, Service de Physique Atomique.

(**) Equipe de Recherches du C. N. R. S. qu'elle implique par des considérations de symétrie. Ils lui ont alors associé la méthode des déphasages généralisés introduits par De Witt [5] et Toulouse [6] pour résoudre complètement le problème des vibrations des surfaces cristallines. Ils ont alors détaillé cette méthode simplifiée, qui est en principe générale, dans le cas d'un cristal monoatomique cubique simple, avec des interactions centrales entre premiers voisins.

Nous nous proposons d'appliquer la méthode des fonctions de Green dans sa forme simplifiée par Dobrzynski et Leman [4] à l'étude des vibrations de la surface (001) du modèle de cristal cubique simple utilisé par Rosenzweig [2] et ceci pour la direction de propagation [100].

Nous retrouvons alors les ondes de Rayleigh à la limite des grandes longueurs d'onde. De plus, nous trouvons des états localisés en surface, dont l'existence a été déjà signalée pour un autre modèle dans la référence [9], à l'intérieur du "gap » $\mathrm{ABC}$ (Fig. 2) délimité par les bandes $\omega_{j}$ du cristal infini. Nous montrons que, dans le cadre de notre modèle de cristal, ces états localisés à l'intérieur du « gap » ABC n'exis- 
tent que sur une partie de l'axe de symétrie $\Delta$ (Fig. 1). Nous trouvons aussi deux "antirésonances" de surface dont l'une correspond à une très faible variation de la densité d'état alors que l'autre correspond à une forte variation de la densité d'état.

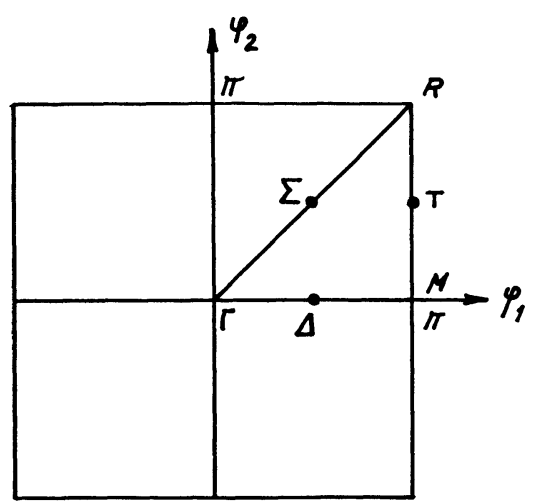

Fig. 1. - Zone de Brillouin à deux dimensions associée à la face (001) d'un cristal cubique simple.

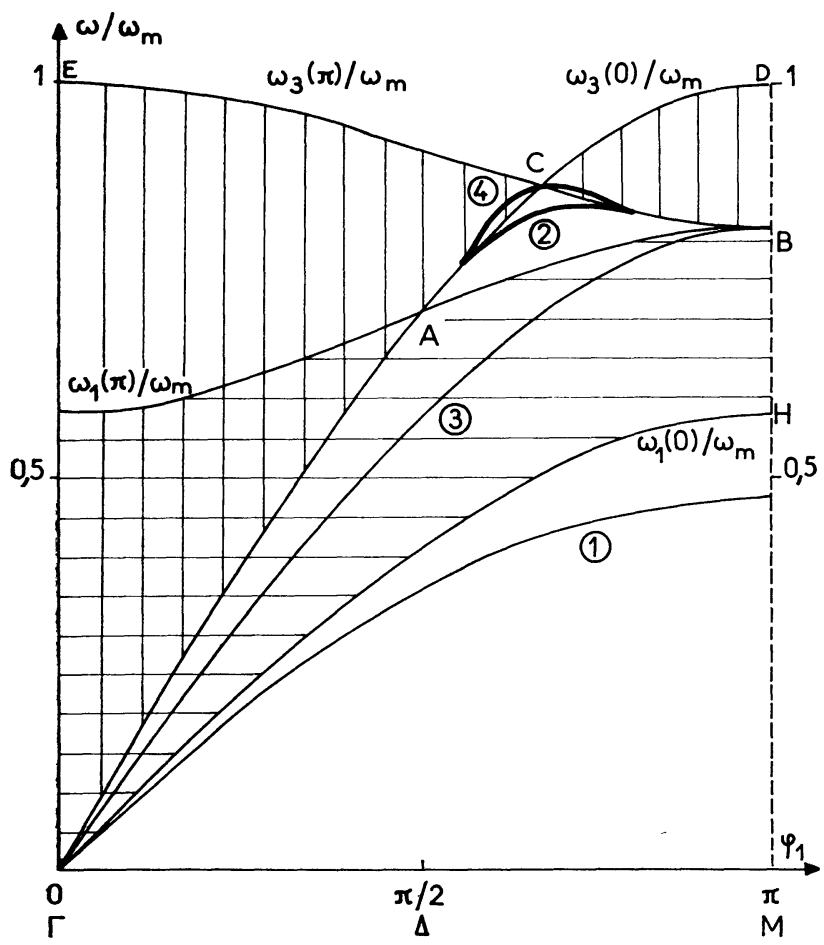

Fig. 2. - Région hachurée : Bandes des fréquences du cristal infini. Courbes 1 et 2 : Etats localisés en surface. Courbes 3 et 4 : "Antirésonances » de surface.

II. Résolution formelle. - Les équations du mouvement indépendantes du temps des atomes d'un cristal s'écrivent, dans l'approximation harmonique, sous la forme matricielle suivante :

$$
\left(D-\omega^{2} I\right) \mathbf{u}=0
$$

où $D$ est la matrice dynamique dont les éléments sont déterminés par les forces qui s'exercent entre les atomes, dans le cadre du modèle de cristal utilisé, $\omega$ est la fréquence angulaire de vibration, $I$ la matrice unité et $\mathbf{u}$ un vecteur colonne composé de $3 N$ éléments donnant les déplacements dans les directions $x, y, z$ des $N$ atomes du cristal par rapport à leur position d'équilibre. La forme des éléments de u dépend des symétries de translation présentées par le cristal étudié.

Nous partons d'un cristal infini cubique simple constitué d'atomes de masses $M$. Soit $D_{0}$ la matrice dynamique qui lui est associée. Nous obtenons un cristal avec deux surfaces libres (cristal perturbé) en coupant le réseau infini associé au cristal non perturbé en deux par le plan $z=a / 2$ et en supprimant les interactions entre les atomes situés de part et d'autre de ce plan. Soit $D$ la matrice dynamique associée au cristal ainsi perturbé.

Nous écrivons $D$ sous la forme :

$$
D=D_{0}+V
$$

où $V$ est la matrice de perturbation.

Nous introduisons les matrices des fonctions de Green $G_{0}^{+}$et $G^{+}$du cristal infini et avec les deux surfaces libres, respectivement :

$$
\begin{aligned}
& G_{0}^{+}=\left(\omega^{2} I-D_{0}+i \varepsilon\right)^{-1} \\
& G^{+}=\left(\omega^{2} I-D+i \varepsilon\right)^{-1}
\end{aligned}
$$

où $\varepsilon$ est infiniment petit positif.

La matrice $G^{+}$s'obtient à partir de $G_{0}^{+}$au moyen de la relation:

$$
G^{+}=\left(I-G_{0}^{+} V\right)^{-1} G_{0}^{+} .
$$

Les fréquences angulaires des états localisés en surface, des résonances et des antirésonances possibles sont les solutions de l'équation :

$$
\operatorname{Re} \operatorname{dét}\left(I-G_{0}^{+} V\right)=0
$$

où $\operatorname{Re} Z$ désigne la partie réelle de $Z$.

La résolution de l'équation (II.6) est simplifiée par le fait que les éléments $V_{n n^{\prime}}^{\sigma \sigma^{\prime}}\left(\varphi_{1}, \varphi_{2}\right)$ de $V$ ne connectent que les plans d'indices $n$ et $n^{\prime}$ parallèles à la surface du cristal et les directions de déplacement $\sigma, \sigma^{\prime}=(x, y$ ou $z)$. Dans ces conditions, il suffit de connaître les éléments $G_{0 n n^{\prime}}^{+\sigma \sigma^{\prime}}\left(\varphi_{1}, \varphi_{2} ; \omega+i \varepsilon\right)$ de $G_{0}^{+}$dont la dimension est la même que celle de $V$.

Connaissant les parties réelles et imaginaires de dét $\left(I-G_{0}^{+} V\right)$, nous pouvons calculer le déphasage généralisé défini par Dewitt [5] et Toulouse [6] :

$$
\eta\left(\omega^{2}\right)=-\operatorname{Arg} \text { dét }\left[I-G_{0}^{+}(\omega+i \varepsilon) V\right] .
$$

La variation de la densité d'états est donnée par :

$$
\Delta n\left(\omega^{2}\right)=\frac{1}{\pi} \frac{\mathrm{d} \eta}{\mathrm{d}\left(\omega^{2}\right)} .
$$

L'étude de la variation de $\eta$ en fonction de $\omega^{2}$ nous permet d'identifier les états de surface, les résonances et les antirésonances possibles [7] :

Rappelons que lorsque la valeur de $\eta$ subit un saut 
brusque de 0 à $+\pi$, nous avons un état localisé en surface à la fréquence angulaire $\omega$ pour laquelle $\eta=+\pi$. A l'intérieur des bandes $\omega_{j}$ du cristal infini, lorsque $\eta$ variant de façon continue en fonction de $\omega^{2}$ passe par $\pi / 2$, nous avons une résonance à la fréquence angulaire correspondante si $\mathrm{d} \eta / \mathrm{d}\left(\omega^{2}\right)>0$ et une « antirésonance " si $\mathrm{d} \eta / \mathrm{d}\left(\omega^{2}\right)<0$. La valeur de $\mathrm{d} \eta / \mathrm{d}\left(\omega^{2}\right)$ à la fréquence angulaire de résonance ou «d'antirésonance » nous renseigne sur l'importance de la variation de la densité d'états à cette fréquence angulaire.

III. Application à un cristal monoatomique cubique simple isotrope à la limite des grandes longueurs d'onde. - Nous utilisons un modèle de cristal cubique simple formé d'atomes identiques de masse $\mathbf{M}$. Nous tenons compte des interactions centrales entre premiers et seconds voisins et aussi des forces dues à la rigidité angulaire d'un système de trois premiers voisins consécutifs formant un angle droit à l'équilibre. Pour ce modèle de cristal, il est possible de satisfaire simultanément les conditions pour la stabilité élastique $\left(c_{11}>0, c_{44}>0, c_{11}+2 c_{12}>0, c_{11}-c_{12}>0\right)$ et les conditions sur les constantes de force venant de ce que l'énergie potentielle doit être invariante pour des rotations infinitésimales de l'ensemble du cristal [8]. Ce modèle a permis à Rosenzweig [2] de retrouver à la limite des grandes longueurs d'onde les ondes de Rayleigh.

III. 1 VALEur PROPRES ET Vecteurs PROPRES DE $D_{0}$ POUR ,L'AXE DE SYMÉTRIE $\Delta$ (Fig. 1). - Les valeurs et vecteurs propres de $D_{0}$ ont été déterminés par Rosenzweig [2] dans tout le plan (101).

On désigne par:

$X_{1}, X_{2}$ : les constantes de forces centrales entre atomes premiers voisins.

$X_{3}$ : la constante de force centrale entre atomes seconds voisins.

$X_{5}$ : la constante de forces dues à la rigidité angulaire d'un système de trois atomes premiers voisins formant un angle droit à l'équilibre.

En choisissant :

$$
X_{1}=2 X_{3} \quad X_{2}=0 \quad X_{3}=X_{5},
$$

Rosenzweig a obtenu sous forme explicite les valeurs et les vecteurs propres de $D_{0}$ :

$$
\begin{aligned}
& \omega_{1}^{2}=\omega_{2}^{2}=\frac{4 X_{3}}{M}\left(2-\cos \varphi_{1}-\cos \varphi_{3}\right) \\
& \omega_{3}^{2}=4 \frac{X_{3}}{M}\left(4-\cos \varphi_{1}-\cos \varphi_{3}-2 \cos \varphi_{1} \cos \varphi_{3}\right) \\
& f_{1}^{x}=\sqrt{\frac{\left(1+\cos \varphi_{1}\right)\left(1-\cos \varphi_{3}\right)}{2\left(1-\cos \varphi_{1} \cos \varphi_{3}\right)} ;} \\
& f_{1}^{y}=0 ; f_{1}^{z}=-\sqrt{\frac{\left(1-\cos \varphi_{1}\right)\left(1+\cos \varphi_{3}\right)}{2\left(1-\cos \varphi_{1} \cos \varphi_{3}\right)}}
\end{aligned}
$$

$$
\begin{gathered}
f_{2}^{x}=0 ; \quad f_{2}^{y}=1 ; \quad f_{2}^{z}=0 \\
f_{3}^{x}=\sqrt{\frac{\left(1-\cos \varphi_{1}\right)\left(1+\cos \varphi_{3}\right)}{2\left(1-\cos \varphi_{1} \cos \varphi_{3}\right)}} \\
f_{3}^{y}=0 ; f_{3}^{z}=\sqrt{\frac{\left(1+\cos \varphi_{1}\right)\left(1-\cos \varphi_{3}\right)}{2\left(1-\cos \varphi_{1} \cos \varphi_{3}\right)}}
\end{gathered}
$$

avec :

$\cos \varphi_{1}=\frac{\mathbf{k} \cdot \mathbf{K}_{\boldsymbol{x}}}{|\mathbf{k}|\left|\mathbf{K}_{\boldsymbol{x}}\right|} ; \cos \varphi_{z}=\frac{\mathbf{k} \cdot \mathbf{K}_{z}}{|\mathbf{k}|\left|\mathbf{K}_{z}\right|}$

où $\quad \mathbf{K}_{x} \equiv[100] ; \mathbf{K}_{z} \equiv[001]$.

Notons que dans ce modèle, les valeurs propres de $D_{0}$ dans le plan (101) sont deux fois dégénérées puisque $\omega_{1}=\omega_{2}$.

Notons que la fréquence maximale de vibration pour ce modèle de cristal est :

$$
\omega_{\mathrm{m}}=\sqrt{24 X_{3} / M} \text {. }
$$

III. 2 MAtrices $V$ ET $G_{0}^{+}$. - Le cristal perturbé que nous étudions est un cristal avec deux surfaces libres (001) obtenues comme nous l'avons indiqué au paragraphe II. En conséquence, seules les équations du mouvement des atomes appartenant à ces deux surfaces diffèreront des équations du mouvement des atomes de volume par des termes, correspondant aux forces supprimées entre les atomes des deux surfaces, qui nous donnent les éléments de la matrice $V$.

Nous calculons analytiquement $G_{0}^{+}$et $V$ pour $\varphi_{2}=0$ En tenant compte, comme dans la référence [4], des symétries que présente notre problème, nous simplifions $G_{0}^{+}$et $V$, ce qui nous permet d'obtenir une expression simple pour dét $\left(I-G_{0}^{+} V\right)$ que nous donnons en annexe.

III. 3 ÉTATS LOCALISÉS EN SURFACE ET « ANTIRÉSONANCES ». - Pour interpréter les résultats de nos calculs, commençons par porter sur la figure 2 les solutions de $\operatorname{Re}$ dét $\left[G_{0}^{+}\right]^{-1}=0$ dans le plan (101), soient $\omega_{1}=\omega_{2}$ et $\omega_{3}$. Pour chaque valeur de $\varphi_{1}, \omega_{1}$ et $\omega_{3}$ sont des fonctions de $\varphi_{3}$. Nous avons fait figurer pour chaque valeur de $\varphi_{1}$ les maxima et minima de $\omega_{1}\left(\varphi_{3}\right) / \omega_{\mathrm{m}}$ et $\omega_{3}\left(\varphi_{3}\right) / \omega_{\mathrm{m}}$. Nous obtenons ainsi quatre courbes qui délimitent les bandes du cristal infini : pour $\omega / \omega_{\mathrm{m}}<\omega_{1}(0) / \omega_{\mathrm{m}}$ nous avons une région interdite. $\mathrm{La}$ région intérieure au triangle $\mathrm{ABC}$ correspond à un "gap » entre $\omega_{1}$ et $\omega_{3}$. La région qui se trouve au-dessus de $\mathrm{CE}$ et $\mathrm{CD}$ est une région interdite.

Nous avons ensuite reporté sur cette même figure les solutions de (II.6).

III. 3.1 Etats localisés en surface. - Nous avons calculé tout au long de l'axe $\Delta$ les états localisés en surface qui correspondent aux ondes de Rayleigh à la limite des grandes longueurs d'onde (Fig. 2, courbe 1). Notons qu'au moyen de l'équation (II.6), dans laquelle nous avons utilisé l'expression du 
dét $\left(I-G_{0}^{+} V\right)$ donnée en annexe, nous avons retrouvé analytiquement, à la limite des grandes longueurs d'onde, les ondes de Rayleigh. Nous trouvons aussi des états localisés en surface à l'intérieur du "gap » ABC (Fig. 2, courbe 2) qui n'existent que pour $102^{\circ} \leqslant \varphi_{1} \leqslant 144$.

III. 3.2 « Antirésonances» de surface. - Nous avons trouvé aussi deux "antirésonances» de surface (Fig. 2, courbes 3 et 4). Nous avons pu déterminer analytiquement la relation de dispersion de «l'antirésonance » (3) en résolvant l'équation (II.6) à l'intérieur de $H \Gamma \mathrm{AB}$ (Fig. 2).

Nous trouvons :

$$
\omega^{2}=8 \frac{X_{3}}{M}\left(1-\cos \varphi_{1}\right)
$$

« L'antirésonance » (3) correspond à une très faible variation de la densité d'état.

« L'antirésonance » (4) n'existe que pour

$$
102^{\circ} \leqslant \varphi_{1} \leqslant 144^{\circ} \text {. }
$$

$$
S=\left[\begin{array}{ccc}
S^{x x} & 0 & S^{x z} \\
0 & 1 & 0 \\
S^{z x} & 0 & S^{z z}
\end{array}\right] \quad A S=\left[\begin{array}{ccc}
(A S)^{x x} & 0 & (A S)^{x z} \\
0 & (A S)^{y y} & 0 \\
(A S)^{z x} & 0 & (A S)^{z z}
\end{array}\right]
$$

avec :

$$
\begin{aligned}
& \begin{array}{l}
S^{x x}=\frac{3\left(1+\cos \varphi_{1}\right)}{2\left(1+2 \cos \varphi_{1}\right)}+\frac{1}{B}\left[\begin{array}{l}
\sin ^{2} \varphi_{1} \sqrt{\left|\omega^{2}-\omega_{3}^{2}(0)\right|\left|\omega^{2}-\omega_{3}^{2}(\pi)\right|}\left(\delta_{3}^{\text {out }}-i \delta_{3}^{\text {in }}\right) \\
\left.-\left(1-\cos \varphi_{1}\right)^{2} \sqrt{\frac{\left|\omega^{2}-\omega_{3}^{2}(\pi)\right|^{3}}{\left|\omega^{2}-\omega_{3}^{2}(0)\right|}\left(\delta_{3}^{\text {out }}+i \delta_{3}^{\text {in }}\right)}\right]
\end{array}\right] \\
S^{z z}=\frac{1-\cos \varphi_{1}}{2\left(1+2 \cos \varphi_{1}\right)}+\frac{1}{B}\left[\begin{array}{l}
4\left(1-\cos \varphi_{1}\right)\left(1+2 \cos \varphi_{1}\right) \sqrt{\left|\omega^{2}-\omega_{1}^{2}(0)\right|\left|\omega^{2}-\omega_{1}^{2}(\pi)\right|}\left(\delta_{1}^{\text {out }}-i \delta_{1}^{\text {in }}\right) \\
+\sin ^{2} \varphi_{1} \sqrt{\left|\omega^{2}-\omega_{3}^{2}(0)\right|\left|\omega^{2}-\omega_{3}^{2}(\pi)\right|\left(\delta_{3}^{\text {out }}-i \delta_{3}^{\text {in }}\right)} \\
-\left(5+\cos \varphi_{1}\right)\left(1+\cos \varphi_{1}\right) \sqrt{\frac{\left|\omega^{2}-\omega_{3}^{2}(0)\right|^{3}}{\left|\omega^{2}-\omega_{3}^{2}(\pi)\right|}\left(\delta_{3}^{\text {out }}+i \delta_{3}^{\text {in }}\right)}
\end{array}\right]
\end{array} \\
& \begin{array}{l}
S^{x x}=\frac{3\left(1+\cos \varphi_{1}\right)}{2\left(1+2 \cos \varphi_{1}\right)}+\frac{1}{B}\left[\begin{array}{l}
\sin ^{2} \varphi_{1} \sqrt{\left|\omega^{2}-\omega_{3}^{2}(0)\right|\left|\omega^{2}-\omega_{3}^{2}(\pi)\right|}\left(\delta_{3}^{\text {out }}-i \delta_{3}^{\text {in }}\right) \\
\left.-\left(1-\cos \varphi_{1}\right)^{2} \sqrt{\frac{\left|\omega^{2}-\omega_{3}^{2}(\pi)\right|^{3}}{\left|\omega^{2}-\omega_{3}^{2}(0)\right|}\left(\delta_{3}^{\text {out }}+i \delta_{3}^{\text {in }}\right)}\right]
\end{array}\right] \\
S^{z z}=\frac{1-\cos \varphi_{1}}{2\left(1+2 \cos \varphi_{1}\right)}+\frac{1}{B}\left[\begin{array}{l}
4\left(1-\cos \varphi_{1}\right)\left(1+2 \cos \varphi_{1}\right) \sqrt{\left|\omega^{2}-\omega_{1}^{2}(0)\right|\left|\omega^{2}-\omega_{1}^{2}(\pi)\right|}\left(\delta_{1}^{\text {out }}-i \delta_{1}^{\text {in }}\right) \\
+\sin ^{2} \varphi_{1} \sqrt{\left|\omega^{2}-\omega_{3}^{2}(0)\right|\left|\omega^{2}-\omega_{3}^{2}(\pi)\right|\left(\delta_{3}^{\text {out }}-i \delta_{3}^{\text {in }}\right)} \\
-\left(5+\cos \varphi_{1}\right)\left(1+\cos \varphi_{1}\right) \sqrt{\frac{\left|\omega^{2}-\omega_{3}^{2}(0)\right|^{3}}{\left|\omega^{2}-\omega_{3}^{2}(\pi)\right|}\left(\delta_{3}^{\text {out }}+i \delta_{3}^{\text {in }}\right)}
\end{array}\right]
\end{array} \\
& S^{x z}=-i \frac{\sin \varphi_{1}}{2\left(1+2 \cos \varphi_{1}\right)}+\frac{\sin \varphi_{1}}{B}\left[\begin{array}{c}
\left(1-\cos \varphi_{1}\right) \sqrt{\left|\omega^{2}-\omega_{3}^{2}(0)\right|\left|\omega^{2}-\omega_{3}^{2}(\pi)\right|}\left(\delta_{3}^{\text {in }}+i \delta_{3}^{\text {out }}\right) \\
+\left(1+\cos \varphi_{1}\right) \sqrt{\frac{\left|\omega^{2}-\omega_{3}^{2}(0)\right|^{3}}{\left|\omega^{2}-\omega_{3}^{2}(\pi)\right|}\left(\delta_{3}^{\text {in }}-\imath \delta_{3}^{\text {out }}\right)}
\end{array}\right] \\
& S^{z x}=i \frac{3 \sin \varphi_{1}}{2\left(1+2 \cos \varphi_{1}\right)}+\frac{\sin \varphi_{1}}{B}\left[\begin{array}{l}
4\left(1+2 \cos \varphi_{1}\right) \sqrt{\left|\omega^{2}-\omega_{1}^{2}(0)\right|\left|\omega^{2}-\omega_{1}^{2}(\pi)\right|}\left(\delta_{1}^{\text {in }}+i \delta_{1}^{\text {out }}\right) \\
-\left(5+\cos \varphi_{1}\right) \sqrt{\left|\omega^{2}-\omega_{3}^{2}(0)\right|\left|\omega^{2}-\omega_{3}^{2}(\pi)\right|}\left(\delta_{3}^{\text {in }}+i \delta_{3}^{\text {out }}\right) \\
-\left(1-\cos \varphi_{1}\right) \sqrt{\frac{\left|\omega^{2}-\omega_{3}^{2}(\pi)\right|^{3}}{\left|\omega^{2}-\omega_{3}^{2}(0)\right|}\left(\delta_{3}^{\text {in }}-i \delta_{3}^{\text {out }}\right)}
\end{array}\right] \\
& (A S)^{x x}=-\frac{1-\cos \varphi_{1}}{2\left(1+2 \cos \varphi_{1}\right)}+\frac{1}{B}\left[\begin{array}{l}
2 \sin ^{2} \varphi_{1} \sqrt{\left|\omega^{2}-\omega_{3}^{2}(0) \| \omega^{2}-\omega_{3}^{2}(\pi)\right|}\left(\delta_{3}^{\text {out }}-i \delta_{3}^{\text {in }}\right) \\
-\left(1+2 \cos \varphi_{1}\right)\left(1+\cos \varphi_{1}\right)^{2} \sqrt{\frac{\left|\omega^{2}-\omega_{1}^{2}(0)\right|^{3}}{\left|\omega^{2}-\omega_{1}^{2}(\pi)\right|}}\left(\delta_{1}^{\text {out }}+i \delta_{1}^{\text {in }}\right) \\
-\sin ^{2} \varphi_{1}\left(1+2 \cos \varphi_{1}\right) \sqrt{\left|\omega^{2}-\omega_{1}^{2}(0) \| \omega^{2}-\omega_{1}^{2}(\pi)\right|}\left(\delta_{1}^{\text {out }}-i \delta_{1}^{\text {in }}\right)
\end{array}\right] \\
& (A S)^{y y}=\sqrt{\frac{\left|\omega^{2}-\omega_{1}^{2}(0)\right|}{\left|\omega^{2}-\omega_{1}^{2}(\pi)\right|}}\left(\left|\delta_{1}^{\text {out }}\right|-i \delta_{1}^{\text {in }}\right)
\end{aligned}
$$

Pour $\varphi_{1} \cong 102^{\circ}$ et $\varphi_{1} \cong 144^{\circ}$ " l'antirésonance " (4) et l'état de surface (2) se rejoignent sur la limite de $\omega_{3}$ du cristal infini. Cette " antirésonance "

Remerciements. - Nous remercions les Professeurs Friedel et G. Leman, le Dr D. A. Degras ainsi qu'ils ont montré pour ce travail ainsi que M. Boiziau

ANNEXE. - Calcul de dét $\left(I-G_{0}^{+} V\right)$. - Comme dans la référence [4] nous avons exprimé la matrice $\left(I-G_{0}^{+} V\right)$ dans la base des fonctions symétriques puisque c'est dans cette base que cette matrice a sa plus simple expression. Nous obtenons :

où $(S)$ et $(A S)$ désignent respectivement les parties symétrique et antisymétrique de $\left(I-G_{0}^{+} V\right)$ : 


$$
\begin{aligned}
& (A S)^{z z}=\frac{1+5 \cos \varphi_{1}}{2\left(1+2 \cos \varphi_{1}\right)}+\frac{1}{B}\left[\begin{array}{l}
2 \sin ^{2} \varphi_{1} \sqrt{\left|\omega^{2}-\omega_{3}^{2}(0) \| \omega^{2}-\omega_{3}^{2}(\pi)\right|}\left(\delta_{3}^{\text {out }}-i \delta_{3}^{\text {in }}\right) \\
-\left(1+2 \cos \varphi_{1}\right)\left(1-\cos \varphi_{1}\right)^{2} \sqrt{\frac{\left|\omega^{2}-\omega_{1}^{2}(\pi)\right|^{3}}{\left|\omega^{2}-\omega_{1}^{2}(0)\right|}}\left(\delta_{1}^{\text {out }}+i \delta_{1}^{\text {in }}\right) \\
-\sin ^{2} \varphi_{1}\left(1+2 \cos \varphi_{1}\right) \sqrt{\left|\omega^{2}-\omega_{1}^{2}(0)\right|\left|\omega^{2}-\omega_{1}^{2}(\pi)\right|}\left(\delta_{1}^{\text {out }}-i \delta_{1}^{\text {in }}\right)
\end{array}\right] \\
& (A S)^{x z}=i \frac{\sin \varphi_{1}}{2\left(1+2 \cos \varphi_{1}\right)}+\frac{\sin \varphi_{1}}{B}\left[\begin{array}{l}
\left(1+\cos \varphi_{1}\right)\left(1+2 \cos \varphi_{1}\right) \sqrt{\left|\omega^{2}-\omega_{1}^{2}(0)\right|\left|\omega^{2}-\omega_{1}^{2}(\pi)\right|}\left(\delta_{1}^{\text {in }}+i \delta_{1}^{\text {out }}\right) \\
-\left(1-\cos \varphi_{1}\right)\left(1+2 \cos \varphi_{1}\right) \sqrt{\frac{\left|\omega^{2}-\omega_{1}^{2}(\pi)\right|^{3}}{\left|\omega^{2}-\omega_{1}^{2}(0)\right|}}\left(\delta_{1}^{\text {in }}-i \delta_{1}^{\text {out }}\right) \\
-2\left(1+\cos \varphi_{1}\right) \sqrt{\left|\omega^{2}-\omega_{3}^{2}(0) \| \omega^{2}-\omega_{3}^{2}(\pi)\right|}\left(\delta_{3}^{\text {in }}+i \delta_{3}^{\text {out }}\right)
\end{array}\right] \\
& (A S)^{2 x}=-i \frac{3 \sin \varphi_{1}}{2\left(1+2 \cos \varphi_{1}\right)}+\frac{\sin \varphi_{1}}{B}\left[\begin{array}{l}
\left(1+\cos \varphi_{1}\right)\left(1+2 \cos \varphi_{1}\right) \sqrt{\frac{\left|\omega^{2}-\omega_{1}^{2}(0)\right|^{3}}{\left|\omega^{2}-\omega_{1}^{2}(\pi)\right|}}\left(\delta_{1}^{\text {in }}-i \delta_{1}^{\text {out }}\right) \\
-\left(1-\cos \varphi_{1}\right)\left(1+2 \cos \varphi_{1}\right) \sqrt{\left|\omega^{2}-\omega_{1}^{2}(0)\right|\left|\omega^{2}-\omega_{1}^{2}(\pi)\right|}\left(\delta_{1 \mathbf{i}}^{\text {in }}+i \delta_{1}^{\text {out }}\right) \\
+2\left(1-\cos \varphi_{1}\right) \sqrt{\left|\omega^{2}-\omega_{3}^{2}(0)\right|\left|\omega^{2}-\omega_{3}^{2}(\pi)\right|}\left(\delta_{3}^{\text {in }}+i \delta_{3}^{\text {out }}\right)
\end{array}\right] \\
& B=4\left(1+2 \cos \varphi_{1}\right)\left[\omega^{2} \cos \varphi_{1}+4 \frac{X}{M^{3}}\left(1-\cos \varphi_{1}\right)^{2}\right] \\
& \omega_{1}^{2}(0)=4 \frac{X_{3}}{M}\left(1-\cos \varphi_{1}\right) \quad \omega_{1}^{2}(\pi)=4 \frac{X_{3}}{M}\left(3-\cos \varphi_{1}\right) \\
& \omega_{3}^{2}(0)=12 \frac{X_{3}}{M}\left(1-\cos \varphi_{1}\right) \quad \omega_{3}^{2}(\pi)=4 \frac{X_{3 \mid}}{M}\left(5+\cos \varphi_{1}\right)
\end{aligned}
$$

$\delta_{j}^{\text {in }}=1$ à l'intérieur de la $j$-ième bande.

$\delta_{j}^{\text {in }}=0$ à l'extérieur de la $j$-ième bande.

$\delta_{j}^{\text {out }}=-1$ au-dessus de la $j$-ième bande.

$\delta_{j}^{\text {out }}=0$ à l'intérieur de la $j$-ième bande.

$\delta_{j}^{\text {out }}=1$ en dessous de la $j$-ième bande.
Les parties symétrique $\eta_{S}$ et antisymétrique $\eta_{A S}$ du déphasage $\eta$ défini en (II.7) s'obtiennent respectivement en calculant les parties réelles et imaginaires des dét $(S)$ et dét $(A S)$ explicitées ci-dessus.

\section{Bibliographie}

[1] Lifshitz (I. M.) et RosenzWeig (L. N.), Izvest. Akad. Nauk S. S. S. R. Série Fiz., 1948, $12,667$.

[2] RosenzWeig (L. N.), Uchenye Zapiski Hark. Gosudarst. Univ. Trudy Fiz. Mat. Otdel., 1950, 2, 19.

[3] Brown (R. A.), Phys. Rev., 1967, 156, 889.

[4] Dobrzynski (L.) et Leman (G.), J. Physique, 1969, 30, 116.
[5] De Witt (B. S.), Phys. Rev., 1956, 103, 1565, Section III.

[6] Toulouse (G.), Solid State Comm., 1966, 4, 593.

[7] Mills (D. L.), J. Physique, Suppl. au no 2, 1967, 28, c-1.

[8] Ludwig (W.) et Lengeler (B.), Solid State Communications, 1964, 2, 83.

[9] Allen (R. E.), Alldredge (G. P.) et de Wette (F. W.), Phys. Rev. Letters, 1969, 23, 1285. 